\title{
Low Cost Database Design for 3d Viewing and Access
}

\author{
Kambiz Farahmand \\ North Dakota State University \\ Kambiz.Farhmand@ndsu.edu
}

\begin{abstract}
Using the popular desktop software, Microsoft Access and Visual Basic coding, a database inventory was designed for a helicopter maintenance plant. This design aims to provide the managers and maintenance engineers a reliable tool to view the inventory list in its shops. This design model uses real time data from the shop floor and the data provided in the Blackhawk Technical Manual for Army operations. This project provides integration between Pro/ENGINEER and Microsoft Access. Microsoft Access is a desktop database application which is inexpensive and available readily. PTC@ Pro/ENGINEER is interactive, powerful parametric modeling software for $3 D$ viewing and modeling. In effect, this design aims to provide the managers with a better understanding and quick access to inventory list of the shop floor and as such better utilization of all the available resources.
\end{abstract}

\section{Introduction}

Databases are designed to offer an organized mechanism for storing, managing and retrieving information in an aircraft industry. Each industry manages database in different format and in different software programs. In general, a Data Base Management System (DBMS) is a computer program, designed to manage a database, a large set of structured data, and run operations on the data requested by numerous users.

At the most basic level, a DBMS is a program that facilitates the storage and retrieval of structured information on a computer's hard drive. A collection of programs that enables you to store, modify, and extract information from a database. There are many different types of DBMSs, ranging from small systems that run on personal computers to huge systems that run on mainframes. Typical examples of DBMS use in different industry include accounting, human resources, and inventory management system and customer support systems. DBMSs have more recently emerged as a fairly standard part of any company back office.
Managing the database in back office has become common in any part of the industry.

This study was focused on design and development of a database inventory for all maintenance tasks of maintenance operation. This led to the utilization of Microsoft Access [1] with Visual Basic coding [2] in the final design of the database. The various models of the aircraft have thousands of parts (data) similar to one another but in different system and subsystem level. Each one of the data is distinguished by different Numbers like National Inventory Number (NIN), National Stock Number (NSN) and also as part number in the Electronic Technical Manual. The data in the Electronic Technical Manual are used in this project and all these data are used to create the database inventory design using Microsoft Access and Visual Basic. Visual Basic coding is used to build the form with the pictures of each part. Microsoft Access is used to build tables, forms and reports. Also Visual Basic coding is used for the search engine.

\section{Technical manual}

The Interactive Electronic Technical Manual is application software, to assist technicians while performing maintenance tasks on the aircraft. This technical manual provides large amounts of information in an easy-to-access format and is also designed to find any mistakes in order to make corrections to improve the existing design. Each technical manual page contains text, figures, part diagrams and search tool to view. These extensive technical manuals provoked this project and get its data values organized at ease. The software tools used here is the Microsoft Access, Visual Basic programming and coding. Microsoft Access is a Relational Data Base Management System (RDBMS), which runs in different version like Microsoft Access 95, 97, 98, 2000, ME, XP.

\section{Gearbox design}


Tail Gearbox is the subsystem of the aircraft's gearbox series system. Each part in the subsystem Tail Gearbox is considered as a data and is put in the table in the first normalization form as per the normalization concept. Figure 1 shows how Gearbox Inventory was designed.

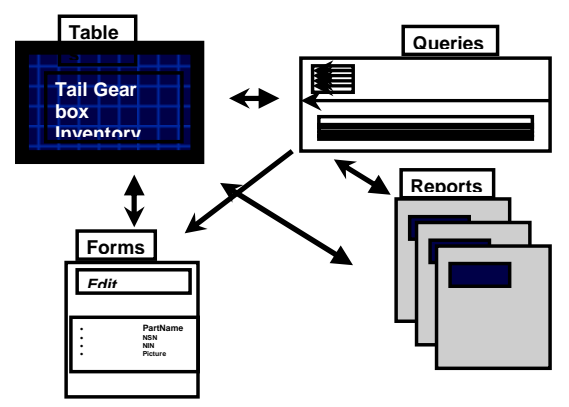

Figure 1. Gearbox inventory design

\section{Programming environment}

Programming Environment used in the project is the Microsoft Access with Visual Basic coding. The database, gearbox inventory uses forms, reports, queries and interfaces. The interfaces used are macro and modules. All these interact with the database value through programs, which is written in Visual Basic. Figure 2 shows the Programming environments in the Microsoft Access.

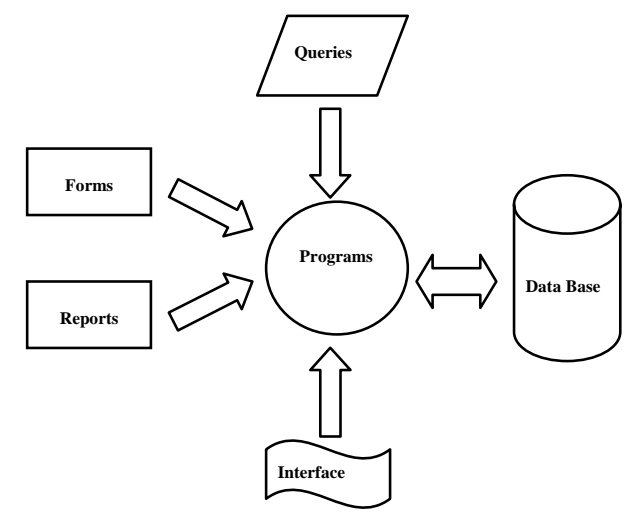
Figure 2. Programming environments in
Microsoft Access

A form is a user-friendly interface to provide user(s) for easier data entry. Data on a form can be made of calculated or combined fields. All these could be done from known fields or from programmatically created fields. Forms are of two types,

1. Input forms

\section{Output forms}

Input forms are dynamic forms so once the input value is submitted its form changes depending on the programs. Figure 3 shows the search operation for searching a part number. As soon as the particular part number is entered and submitted, the search is initiated for the particular data parameter and the form changes dynamically. Figure 4 is static but it also run with VB programs which switches to another static form. Output forms are mainly used in the report generation of the database.

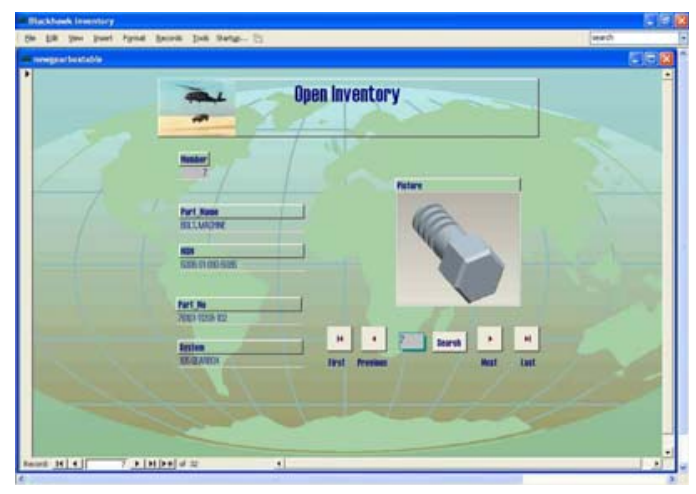

Figure 3. Input form

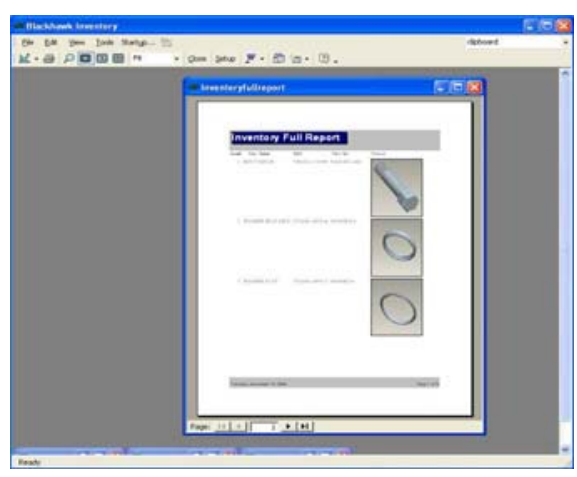

Figure 4. Output form

Front start-up form is designed with Microsoft Access and Visual Basic coding. This presents the user agreement and copyright information. The form is set by a timer to pause for 10 seconds so that the user can view the agreement and copyright. The output form in Figure 5 is the main inventory form which contains buttons for opening inventory, editing inventory, searching inventory and getting reports for the inventory. It also has the exit button to exit the application and close the database and exit the application. 


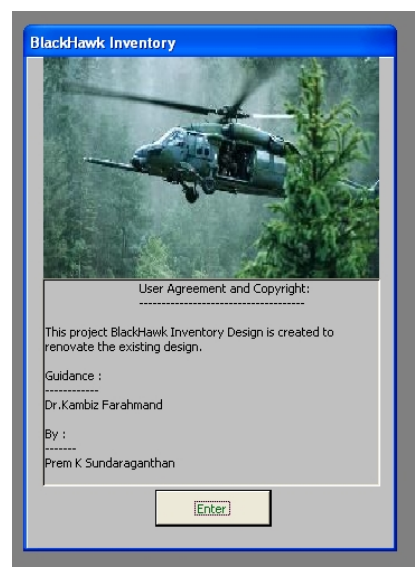

Figure 5. Main inventory form

Figure 6 shows the open inventory form where all the Part Name, National Stock Number, Part Number, and System Name. Navigation buttons are used for moving to next, previous, first, last records of data. Thus entering the number and pressing the search button can obtain particular record.

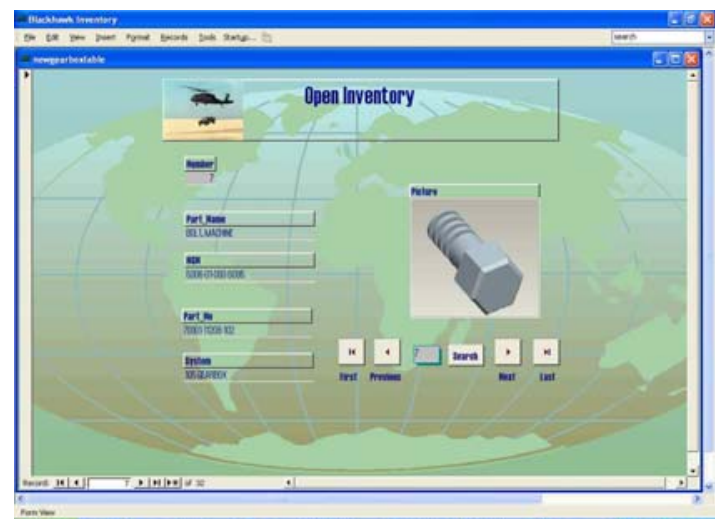

Figure 6. Open inventory form

Figure 7 shows the edit inventory form. Edit inventory form has sub-form like add, update, delete inventory form. These forms perform all the basic operations such as add inventory, update inventory, delete inventory, etc. There is a back button to go back to the previous form and also an exit button to exit the database and the application.

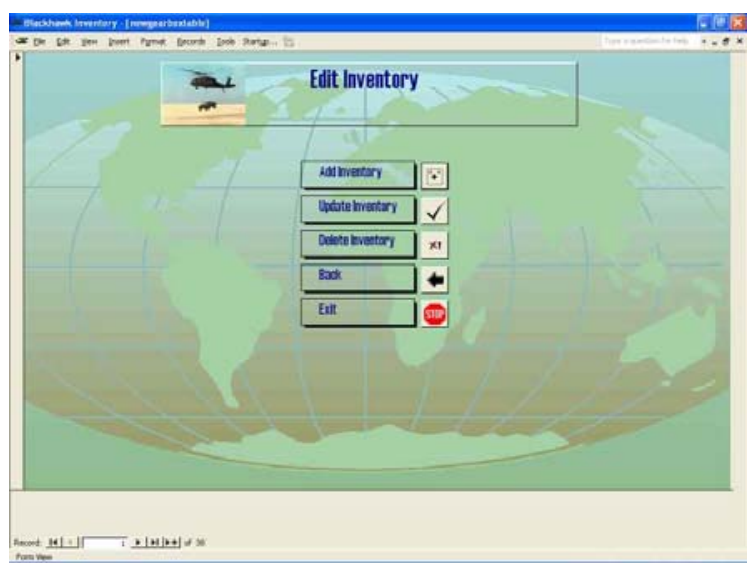

Figure 7. Edit inventory form

Figure 8 shows the report inventory form. Report inventory form has sub-form such as inventory report, part name report, NSN report which makes printing on selected categories easier. There is a back button to go back to the previous form and also an exit button to exit the database and the application.

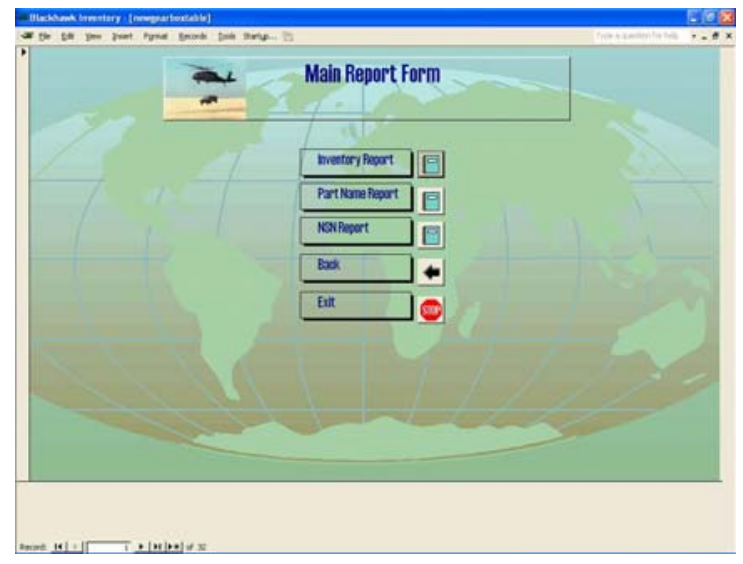

Figure 8. Report inventory form

Figure 9 shows the search inventory form. Search inventory form has sub-form such as search by number search by part name, search by part number and search by NSN which makes queries on selected categories to be seen and sorted out. There is a back button to go back to the previous form and also an exit button to exit the database and the application. 


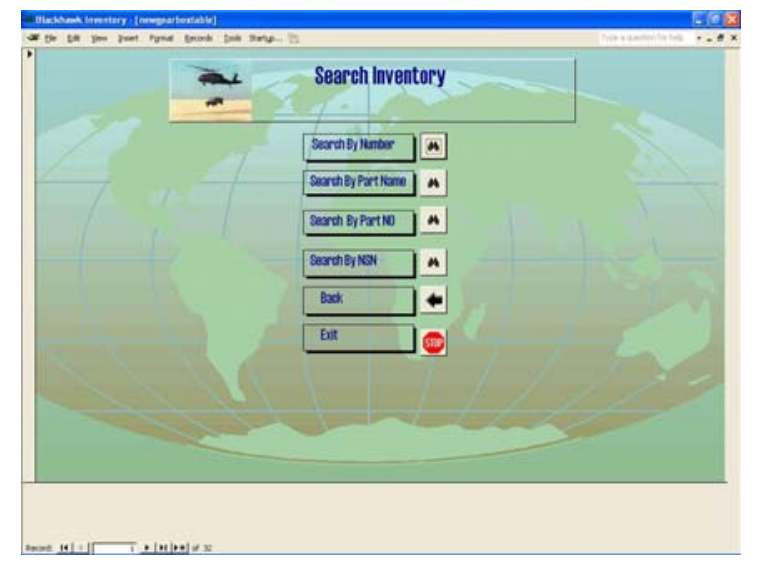

Figure 9. Search inventory form

A report shown in Figure 10, is an effective way to present data in a printed format. There is adequate control over the size and appearance of all parameters on a report including how it is presented.

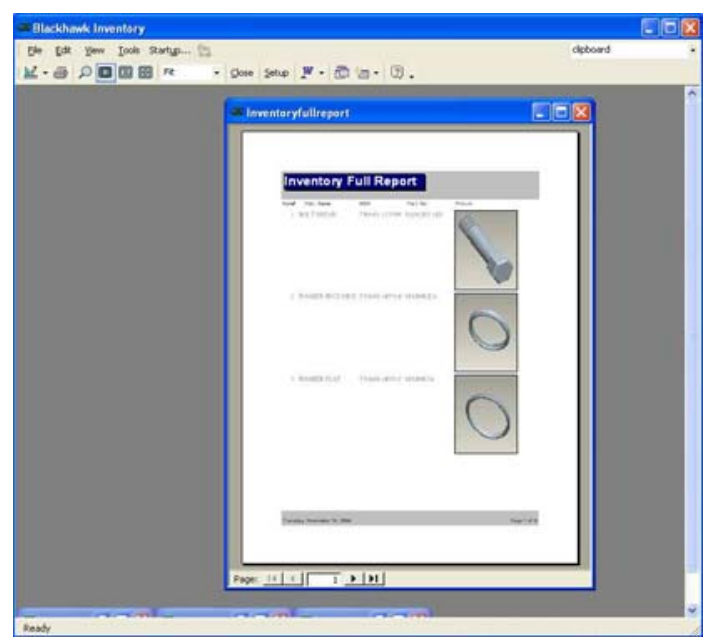

Figure 10. Report form

A database generated Table is depicted in Figure 11. A table is a collection of data about a specific topic, such as parts, vendors, or contacts. Using a separate table for each topic means that we store that data only once, which makes the database more efficient, and reduces data-entry errors. Tables can be created in the design view or in the database table wizard. Data types must be specified before tables are created.

\section{Object linking and embedding}

Object Linking and Embedding (OLE) is a distributed object system and protocol from Microsoft Corporation. For example, a desktop publishing system might send some text to a word processor or a picture to a bitmap editor using OLE.

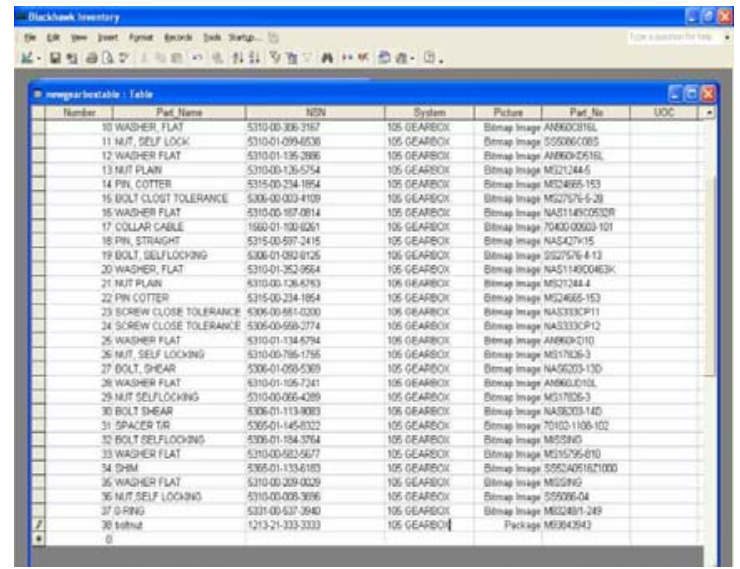

Figure 11. Inventory table

In this project OLE object is used to import pictures into the database and to make it as a data value. An OLE server is the application that creates OLE objects. The objects can be embedded or linked in an OLE container application. An OLE container displays OLE objects. An application can be an OLE server application, OLE container application, or both. Microsoft Paint, Microsoft Word, and Microsoft Excel, AutoCAD, Pro-E are examples of OLE server applications. In this project, object is linked and also embedded. An OLE object is embedded or linked in an OLE container, which is simply an item in Form or in Table. Embedded objects become part of the Forms module, and linked objects are references from a form module to a linked source file. A linked object is a reference from the form to a file. Updating the data in the OLE Container will update the file if the changes are saved.

\section{Pro/ENGINEER integration with Microsoft Access}

Object Linking \& Embedding is done with the PTC ${ }^{\odot}$ software, Pro/ENGINEER ${ }^{\circledR}$ to give 3D view of the part diagram. OLE server of MS-Access and OLE server of $\mathrm{PTC}^{\odot}$ software is made compatible and is being communicated to share the image of the part drawings. Figure 12 shows that the Microsoft Access OLE Server is made to communicate with PTC $^{\circ}$ OLE server. 


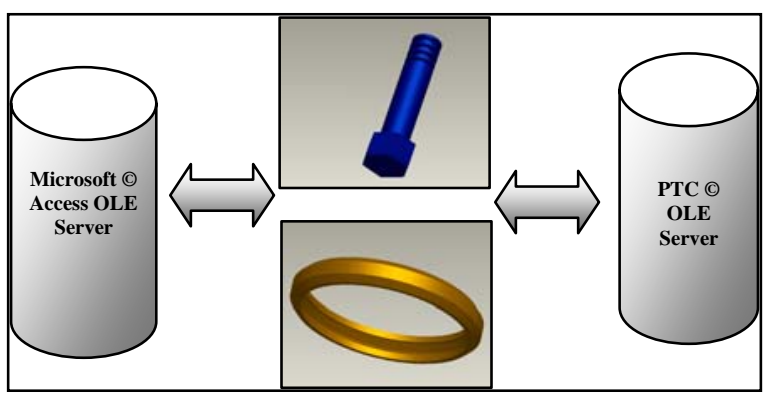

Figure 12. Pro/ENGINEER $®$ integration with Microsoft Access

\section{PTC@ OLE server}

While Installing Pro/ENGINEER ${ }^{\circledR}$ Training or wildfire Edition, the OLE setting has to be adjusted. By keeping the default OLE settings box checked on the Optional Configuration Steps window you can configure Pro/ENGINEER ${ }^{\circledR}$ Training or wildfire Edition as an OLE (Object Linking and Embedding) Server. This allows the user of the client application to import Pro/ENGINEER ${ }^{\circledR}$ files (parts, assemblies, and drawings) into Microsoft documents (Access, Word, Excel, and Power Point). Figure 13 shows the PTC ${ }^{\odot}$ OLE Server.

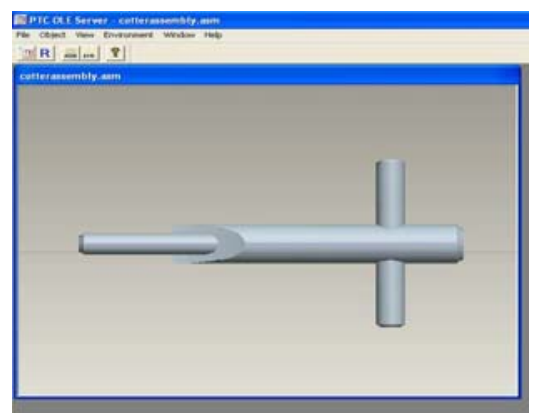

\section{Figure 13. PTC $\odot$ OLE server with assembly diagram}

PTC $^{\odot}$ OLE server is embedded as a menu bar in the Database Inventory design software. This helps the user to navigate between different viewing options of Pro/ENGINEER ${ }^{\circledR}$ package. From this user can view the part or assembly diagram with two options, one with Pro/ENGINEER wildfire ${ }^{\circledR}$ package editor and other with the PTC ${ }^{\odot}$ Product view express. Figure 14 shows how the PTC $^{\odot}$ OLE server is embedded as a menu bar in the Database Inventory design software.

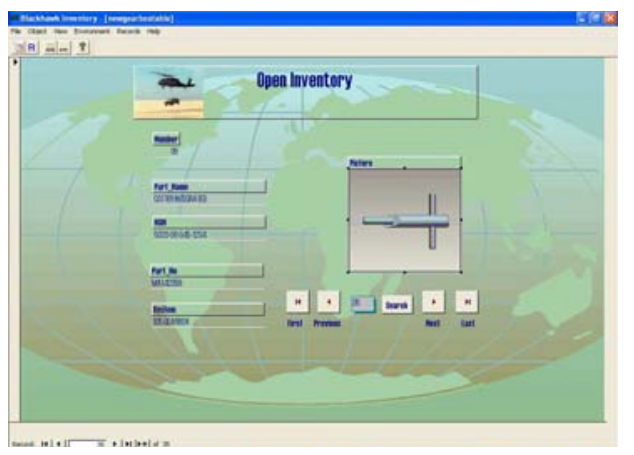

\section{Figure 14. PTC $\odot$ OLE toolbar in database inventory software}

\section{Conclusion}

This study brings a better desktop interactive technical manual to the shop floor. Data and information are accessed quickly and inexpensively. This project consists of front end implemented using Visual Basic 6.0 and a backend using Microsoft Access XP. Visual Basic utilizes different forms to connect to the Access database for different operations like opening, viewing, appending, updating and deleting from the database. The search engine, which was incorporated, uses a heuristic search algorithm to query and retrieve Part Number, National Stock Number, and Part Name. The Backend uses query processing to integrate common fields in the database where constraints allow excellent maneuverability between forms and their corresponding tables. Security enhancement for different levels of query abstraction has also been implemented in the backend. Furthermore, a report generator with a preview function has also been included in the backend module. This project brings successful integration between PTC $^{\odot}$ Pro/ENGINEER wildfire ${ }^{\circledR}$ and also Microsoft Access through the Object Linking and Embedding (OLE) concept. This can be extended for different 3D software in market, because since most of the software is based on Object concept. AutoDesk ${ }^{(}$ AutoCAD different versions can bring 3D \& 2D part drawings inside this software.

\section{References}

[1]. Http://www.fgcu.edu/support/office2000/access/ Good source for MS-Access.

[2]. Francesco, B., "Programming Microsoft Visual Basic 6.0”, Microsoft Press, June 1999. 